\title{
Cost Analysis of Algorithm Based Billboard Manger Based Handover Method in LEO satellite Networks
}

\author{
Suman Kumar Sikdar \\ Department of Computer science \& Engineering, \\ University of Kalyani \\ Kalyani-741235, India
}

\author{
Soumaya Das \\ Department of E.T.C.E Bengal Institute of technology, \\ West Bengal University of technology \\ Kolkata, India
}

\author{
DebabrataSarddar \\ (Astt Professor) Department of Computer science \\ \& Engineering, University of Kalyani, \\ Kalyani-741235, India
}

\begin{abstract}
Now-a-days LEO satellites have an important role in global communication system. They have some advantages like low power requirement and low end-to-end delay, more efficient frequency spectrum utilization between satellites and spot beams over GEO and MEO. So in future they can be used as a replacement of modern terrestrial wireless networks. But the handover occurrence is more due to the speed of the LEOs. Different protocol has been proposed for a successful handover among which BMBHO is more efficient. But it had a problem during the selection of the mobile node during handover. In our previous work we have proposed an algorithm so that the connection can be established easily with the appropriate satellite. In this paper we will evaluate the mobility management cost of Algorithm based Billboard Manager Based Handover method (BMBHO). A simulation result shows that the cost is lower than the cost of Mobile IP of SeaHO-LEO and PatHOLEO.
\end{abstract}

Keywords-Component; Handover latency; LEO satellite; Mobile Node (MN); Billboard Manager (BM).

\section{INTRODUCTION}

Modern terrestrial networks are designed as per to give the low cost and best quality service. Mobile networks provide communication to a limited geographical area. The applications of satellite network increases in order to provide the global coverage in a large area. There are different satellites for this communication [1] [2].

1. Geostationary satellite

2. Medium Earth Orbit satellite

3. Low Earth Orbit satellite.

Among which Low Earth Orbit satellite is the best for communication and as the replacement of future terrestrial wireless networks as it has some advantages like
a. Low propagation delay
b. Low end to end delay
c. Low power requirement

d. More efficient frequency spectrum utilization between satellites and spot-beams.

But the Leo satellite has also some problems. The main problem is that the low earth orbit satellites have a large relative speed than the speed of mobile nodes (MN) \& earth. That's why the handover occurrence is more. So the call blocking probability $\left(\mathrm{P}_{\mathrm{b}}\right)$ and force call termination probability (Pf) is also higher. To solve this problem different handover techniques have been proposed.

\section{What is handover?}

Handover is the process of transferring satellite control responsibility from one earth station to another earth station without any loss or interruption of the service[3][4].

An unsuccessful handover can degrade the system performance like call quality as well as it call because the forced call termination. To solve these problems different handover technique has been proposed [5] [6]. A handover is done in the following three steps:
i. $\quad$ Scanning
ii. Authentication
iii. Re-association

Scanning: When a mobile station is moving away from its current satellite, it initiates the handoff process when the received signal strength and signal-to-noise-ratio have decreased below the threshold level. The MN now begins the scanning to find new satellite. It can either go for a passive scan (where it listens for beacon frames periodically sent out by satellites) or choose a faster active scanning mechanism wherein it regularly sends out probe request frames and waits for responses for $\mathrm{T}_{\text {MIN }}$ (min Channel Time) and continues scanning until TMAX (max Channel Time) if at least one response has been heard within $\mathrm{T}_{\mathrm{MIN}}$. Thus, $\mathrm{n}^{*} \mathrm{~T}_{\mathrm{MIN}} \leq$ time to scan $n$ channels $\leq n^{*} T_{\text {MAX. }}$ The information gathered is then processed so that the MN can decide which Satellite to join next. The total time required until this point constitutes $90 \%$ of the handoff delay. 
Authentication: To associate the link with the new satellite Authentication is necessary. Authentication must either immediately proceed to association or must immediately follow a channel scan cycle. In pre-authentication schemes, the $\mathrm{MN}$ authenticates with the new satellite immediately after the scan cycle finishes.

Re-Association: Re-association is a process for transferring associations from old satellite to new one. Once the $\mathrm{MN}$ has been authenticated with the new satellite, reassociation can be started. Previous works has shown reassociation delay to be around 1-2 ms. The range of scanning delay is given by:-

\section{$\mathrm{N} \times \mathrm{Tmin}_{-}$Tscan _ $\mathrm{N} \times \mathrm{Tmax}$}

Where $\mathrm{N}$ is the total number of channels according to the spectrum released by a country, Tmin is Min Channel Time, Tscan is the total measured scanning delay, and Tmax is Max Channel Time. Here we focus on reducing the scanning delay by minimizing the total number of scans performed.

The paper is organized as follows: In the second section we have described the related works on handover management including Mobile IP, SeaHO-LEO, PatHO-LEO and our previously proposed Algorithm Based BMBHO. In the third section we have described the proposed work which includes the cost analysis of Algorithm Based BMBHO. In the forth section the simulation results of both our method and standard methods. In the section 5 we conclude the whole paper and finally a future work is mention regarding this paper in section six.

\section{RELATED WORK:}

To solve the problems of unsuccessful handovers different handover management protocols have been proposed [7] .The most widely used one is MIP i.e. Mobile IP Network [8]. It is proposed by The Internet engineering task force (IETF) to handle mobility of internet hosts for mobile data communications. MIP is based over the concept of Home Agent (HA) and Foreign Agent (FA) for delivering of packets from one $\mathrm{MN}$ to $\mathrm{CN}$.

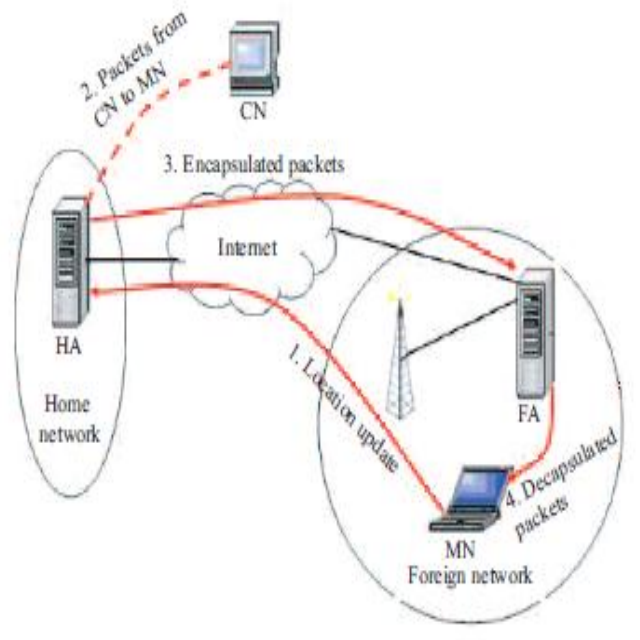

Figure 1: Handover scenario in MIPIt is done in four steps. a. At the beginning of the handover $\mathrm{MN}$ registers itself in FA and waits for the channel allocation in FA and also updates its location in HA directory.

b. Then the packets are sent to HA and HA encapsulate it.

c. After that encapsulated packets are sent to The FA. MN.

d. Lastly FA decapsulate those packets and sent it to

But this protocol also has some drawbacks. The main drawbacks of this protocol are:

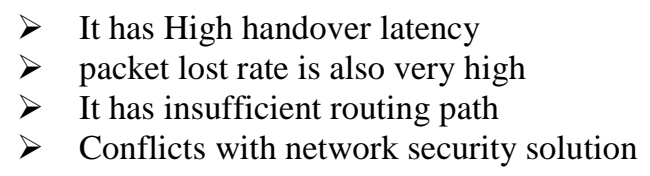

So to overcome this drawbacks another protocol have been proposed i.e. Seamless handover management scheme (SEAHO-LEO) [9] [10] [11].

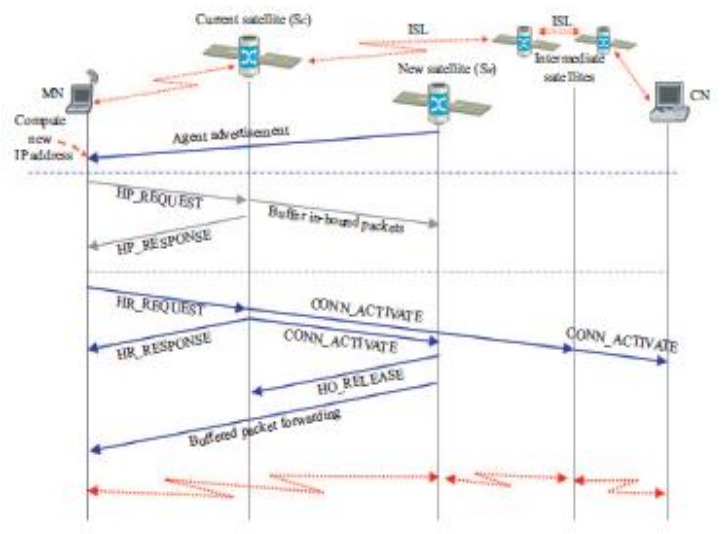

Figure 2: Handover scenario in SeaHO-LEO

This can be done in the following steps:

A. Calculate a new IP

B. Send handover preparation request to current satellite

C. Start to use new IP to send data packets

D. CN starts to use new satellite

The main disadvantage of this process is

$>$ packet loss is less

$>$ It has lower handover latency.

The main disadvantage of this process is

High messaging traffic.

To get over these drawbacks another method to remove high messaging traffic is Pattern based handover management (PatHO-LEO) [8], [9].

It describes as follows

$>$ Satellite register to BM.

$>\mathrm{MN}$ registers to $\mathrm{BM}$.

$>\mathrm{BM}$ establishes the satellite and user mobility pattern (SMUP) table.

$>\mathrm{CN}$ and $\mathrm{BM}$ establish connection.

$>\mathrm{CN}$ sends data packets to MN.

But the main drawback of PatHO-LEO is that 
Every user should have a specific mobility pattern in a specific period of time. A user can have more than one mobility pattern. But when it violets its mobility pattern the handover process will be either in SeaHO-LEO or MIP.

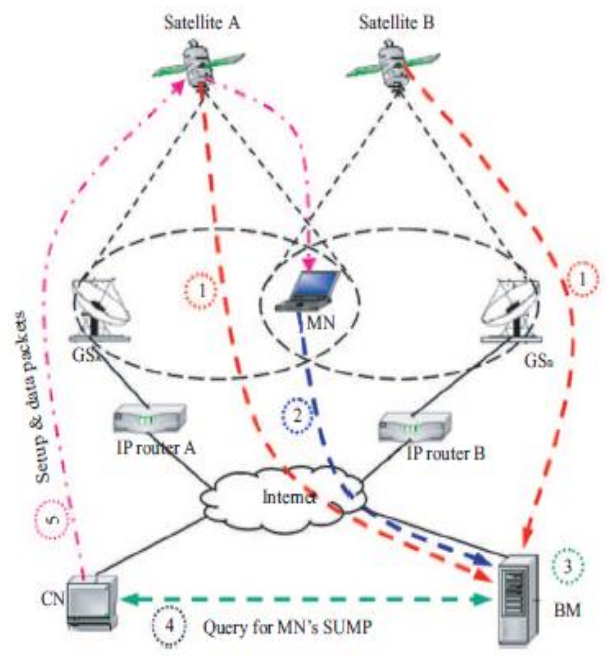

Figure 3: Handover scenario in PatHO-LEO

The no of user who do not have a specific mobility pattern in a week is increasing day by day like salesman, LIC worker who have to go different place at different time in a week.

Now to overcome these existing problems the handover management protocol proposed is named as BMBHO i.e. Billboard Manager Based Handover Technique where BM only stores satellite location signal strength based on QoS parameters[12][13].

* In BMBHO we assume that the direction of the signal flow is in one side or both side i.e. from $\mathrm{CN}$ to $\mathrm{MN}$ (where $\mathrm{CN}$ is fixed \& MN is movable) or from MN1 to MN2 and vice versa (where both are movable).

* If the CN/MN2 is under the footprint of same satellite then the communication will be via one satellite otherwise via different satellite by ISL. So it is not important to know that communication is through $\mathrm{CN} / \mathrm{MN} 2$, as the method is same for all.

This handover process can be done in the following ways

1) BM stores all info about satellites: the entire satellites resister to BM including their IP address. This information not subjected to change and permanently stored in the BM database.

2) All satellite sends periodic info: All the satellites will send the following info periodically to the BM.

i) Channel capacity: -- How many channels are available in the satellite.

ii) Signal strength: --What is the strength of the signal at that time because from time to time and area to area due to the different weather condition.

This information is not constant $\&$ it updates itself every time it gets a new info. The time period of this update will be set as small as possible because a huge no of MN lies under the footprint of a satellite. So the channel capacity changes very frequent. This time period is inversely proportional to the success of handover.

3)Handover request is send to BM: If a new $\mathrm{MN}$ wants to handover i.e. its signal strength decreases under a certain level called threshold level, it sends a HANDOVER_REQUEST (HO_REQ) to BM via its current satellite which contains the following

i) IP addresses of the current satellite (CS),

ii) IP address of adjacent satellite (AS) If MN/MN1 is connected to $\mathrm{CN} / \mathrm{MN} 2$ through more than one satellite by ISLs.

iii) IP address of MN itself.

ii) Position of MN.

iii) The direction of the $\mathrm{MN}$ i.e. in which direction it wants to go.

4)BM selects the new satellite: Now BM first makes a list of available satellites in that direction at that time with the help of its stored data \& the updates of satellites. Then BM selects best satellites for that $\mathrm{MN}$ according to the QoS parameters. A specific algorithm has to be developed for selecting the correct satellite.

5)MNstarts to use new satellite: Once the satellite is selected BM sends the IP address of the new satellite to the MN \& CN/MN2/AS. Now CN/MN2/AS makes a connection set up for the new satellite and it communicates to $\mathrm{MN}$ via the new satellite.

\section{ALGORITHM:}

The algorithm of BMBHO [14] is as follows

1) BM stores all information about satellite like IP addresses of the satellite.

2) All satellite sends periodic information to Billboard Manager.

a) Channel capacity

b) Signal strength

Both of the information varies time to time and also area to area.

3) Now for $t=0$,compare channel capacity if the channel capacity $>0$

Continue;

Else stop

4) Compare channel capacity, choose the maximum one.

5) If the channel capacity of the two satellite to handover is same,

6) Compare the signal strength. Choose the lowest signal strength of same channel capacity.

Else go back to 4

7) Repeat 4-6 every time while choosing a new satellite to handover.

8) Make a list of the available satellites and store it to BM

9) Now, If a new mobile node wants to handover, signal strength decreases under a certain level i.e. threshold level, it 
sends a Handover Request to $\mathrm{BM}$ via its current satellite containing

a) IP address of the current satellite.

b) IP address of the adjacent satellite, If MN/MN1 is connected to CN/MN2 through more than one satellite by ISLs.
c) IP address of $\mathrm{MN}$.
d) Position of $\mathrm{MN}$
e) The direction of the $\mathrm{MN}$

10) Now BM again makes a list of available MNs.

11) Now comparing the first list and second list it chooses the best satellite to handover.

12) Once the satellite is selected, BM sends $M N$ the IP address of the new satellite.

13) Now the connection is established.

\section{FLOW CHART:}

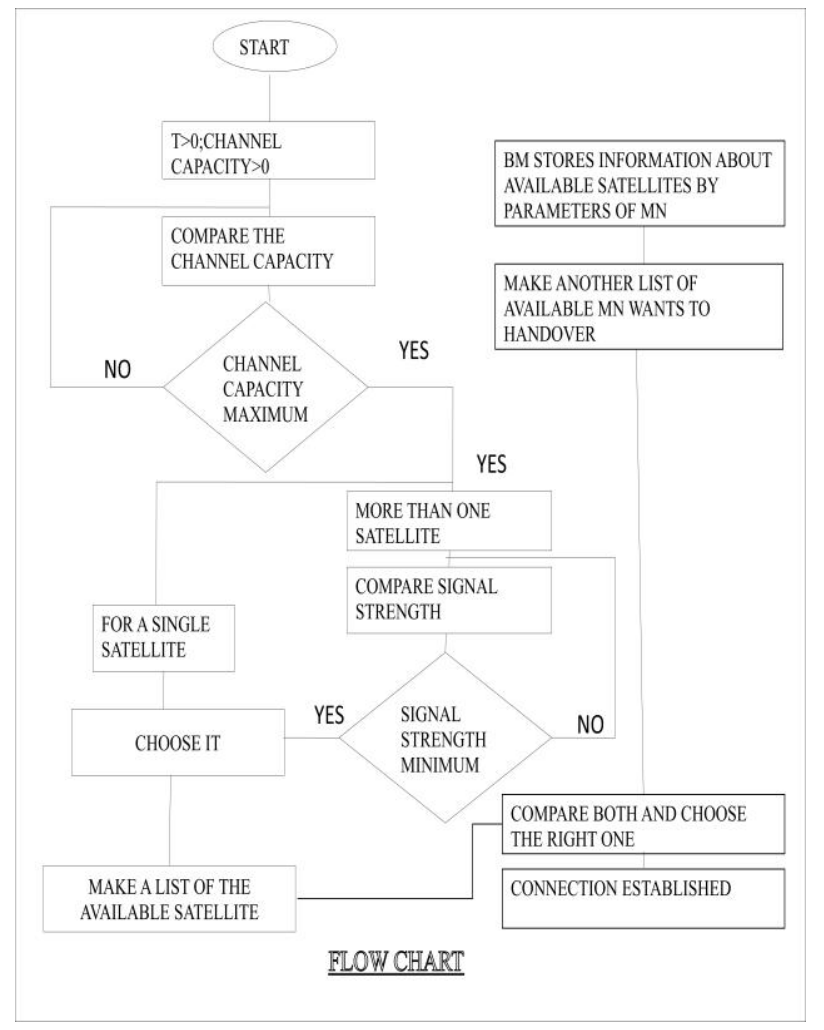

Figure 4: Algorithm for establishing connection

\section{PROPOSED WORK:}

In our previous work we have discussed the Algorithm based BMBHO method and shown how it can reduce handover latency and call blocking probability.

Here we will analysis the cost of this Algorithm based BMBHO method and compare it with other standard methods like MIP.

\section{Mobility Management Cost Definition}

In [12] the mobility management cost is evaluated as the product of generated control message size, $\mathrm{M}$ and the number of hopes, $\mathrm{H}$, required to deliver the message. If we apply such definition into the paging cost, it will be proportional with the number of receivers. Taking into account the broadcasting capabilities of satellites, however, the cost is also simply a product of the message size and the number of travelled hops.

Cost=M.H

(1)

\section{Costs of different Mobility management events:}

The following defines the cost required for each mobility management event; binding update, local forwarding and paging

For each case, the Control messages generated are assumed to be equally sized (M) in all the four events.

The number of control messages that are generated upon a handover occurrence between mobile nodes and the corresponding ARs, is assumed to be same for MIP and our proposed method. Thus we can neglect the number of control message in the cost evaluation.

1. Binding Update Cost: Let $H_{M N, L D}$ denote the number of hops between a mobile node and the Location Directory. The cost for binding update procedure can be expressed as:

$$
\text { M. } \mathrm{H}_{\mathrm{MN}, \mathrm{LD}}
$$

2. Local Forwarding Cost: Denoting the number of hops between two adjacent satellites as $H_{A R, A R}$ the local forwarding cost is shown as follows:

$$
\mathrm{M} \cdot \mathrm{H}_{\mathrm{AR}, \mathrm{AR}}
$$

\section{Management Cost of MIP and our proposed method}

The costs of Mobile IP and our proposed method are as follows

A. Mobile IP: The cost of MIP is the product of binding update cost and rate of handover occurrence. The local forwarding, paging and GPS are not used here. So the MIP management cost, $\mathrm{C}_{\mathrm{MIP}}(\mathrm{t})$ can be expressed as

$$
\mathrm{C}_{\mathrm{MIP}}(\mathrm{t})=\mathrm{M} \cdot \mathrm{H}_{\mathrm{MN}, \mathrm{LD}} \cdot \mathrm{R}_{\mathrm{HO}}(\mathrm{t})
$$

Where the rate of handover occurrence, $R_{H O}(t)$, is:

$$
\mathrm{R}_{\mathrm{HO}}(\mathrm{t})=\mathrm{V}_{\text {sat }} \cdot \mathrm{L}_{\mathrm{sat}} \int_{V s a t(t-t \Delta)}^{V s a t . t} D L(\text { Vsat. } t) d t
$$

Where, $\mathrm{V}_{\text {sat }}$ and $\mathrm{L}_{\mathrm{sat}}$ denote the ground speed of satellite and the coverage boundary length, respectively. $D_{L}\left(V_{\text {sat }} \cdot t\right)$ denote the linear density of nodes on the coverage boundary at time $t$.

B. PatHO-LEO: In the PatHO-LEO model, the local forwarding and paging scheme create some additional cost. The total cost of PatHO-LEO model $\mathrm{C}_{\text {PatHO-LEO }}(\mathrm{t})$ is

$$
\mathrm{C}_{\text {PatHO-LEO }}(\mathrm{t})=\mathrm{M} \cdot \mathrm{H}_{\mathrm{MN}, \mathrm{LD}}+\mathrm{M} \cdot \mathrm{H}_{\mathrm{AR}, \mathrm{AR}} \mathrm{R}_{\mathrm{HO}}(\mathrm{t}) \cdot \alpha
$$
$\left.\left\{\mathrm{M}_{\mathrm{H}, \mathrm{H}}(\mathrm{S}-1)+\mathrm{M} . \mathrm{S}\right\}\right\}^{*} \mathrm{n}(\mathrm{t})(1-\alpha) \cdot \lambda$

Where,. $\mathrm{H}_{\mathrm{AR}, \mathrm{AR}}$ and $\mathrm{S}$ denote the number of hops between two adjacent satellites and the number of single-beam satellites that cover a single paging area, respectively. $\mathrm{n}(\mathrm{t})$ and $\alpha$ denote the total number of MNs per a coverage area at time $t$ and the ratio of active $\mathrm{MNs}$ to the total number of $\mathrm{MNs}$, respectively. The rate of new connections to a $\mathrm{MN}$ is denoted as $\lambda$.

C. Proposed Work: In our proposed method we have introduced the billboard manager (BM) so that we have 
successfully removed the scanning cost but it will introduce the messaging cost which we will evaluate now

1. Messaging Cost in a day: As all channel sends periodically the messages to $\mathrm{BM}$ which contains two information channel capacity and signal strength so if $\mathrm{N}$ be the total number of satellite and $\mathrm{t}$ (in sec) will be the time interval between sending two successive messages so the total number of messaging in a day will be

$$
\mathrm{C}_{\mathrm{MSG}, \mathrm{DAY}}=24 *\left\{\left(\mathrm{M} . \mathrm{H}_{\mathrm{Sat}, \mathrm{BM}} * 3600\right) / \mathrm{t}\right\} * \mathrm{n}
$$

2. Messaging Cost per handover: Now we will evaluate the messaging cost per handover. When the signal strength and signal to noise ratio decreases below the threshold level then every MN sends the handover request HR_REQ and after performing the BMBHO algorithm BM sends IP of the current satellite and adjacent satellite to $\mathrm{MN}$ so the required messaging cost between $\mathrm{MN}$ and $\mathrm{BM} \mathrm{C}_{\mathrm{MN}, \mathrm{BM}}$ is

$$
\mathrm{C}_{\mathrm{MN}, \mathrm{BM}}=2 * \mathrm{M}^{*} \mathrm{H}_{\mathrm{MN}, \mathrm{BM}}(6)
$$

Now the message transfer between satellites to BM is also two which contains the IP address of the MN; one to the current satellite and another to the adjacent satellite. So the total messaging cost between $\mathrm{BM}$ and satellites $\mathrm{C}_{\text {Sat,BM }}$ is

$$
\mathrm{C}_{\mathrm{Sat}, \mathrm{BM}}=\mathrm{M}^{*}\left(\mathrm{H}_{\mathrm{CSat}, \mathrm{BM}}+\mathrm{H}_{\mathrm{AdSat}, \mathrm{BM}}\right)
$$

Where $\mathrm{H}_{\mathrm{CSat}, \mathrm{BM}}$ is the message transfer between the current satellite and $\mathrm{BM}$ and $\mathrm{H}_{\mathrm{AdSat}, \mathrm{BM}}$ is the message transfer between adjacent satellite and BM.

Now if the handover involves only one satellite then

$$
\mathrm{H}_{\mathrm{AdSat}, \mathrm{BM}}=0
$$

Let $\mathrm{K}$ be the total number of handover in a day and $\mathrm{L}$ be the total number of handover which involves only one satellite then equation 7 reduces to

$$
\mathrm{C}_{\mathrm{Sat}, \mathrm{BM}}=\mathrm{M}^{*}\left\{\mathrm{H}_{\mathrm{CSat}, \mathrm{BM}} *(\mathrm{~K}-\mathrm{L})+\mathrm{H}_{\mathrm{AdSat}, \mathrm{BM}} * \mathrm{~L}\right)
$$

So now the total messaging cost for handover $\mathrm{C}_{\mathrm{MSG}, \mathrm{HO}}$ is

$$
\begin{aligned}
& \mathrm{C}_{\mathrm{MSG}, \mathrm{HO}}=\mathrm{C}_{\mathrm{MN}, \mathrm{BM}}+\mathrm{C}_{\mathrm{Sat}, \mathrm{BM}} \\
& =2 * \mathrm{M} * \mathrm{H}_{\mathrm{MN}, \mathrm{BM}}+\quad \mathrm{M} *\left\{\mathrm{H}_{\mathrm{CSat}, \mathrm{BM}} *(\mathrm{~K}-\mathrm{L})+\mathrm{H}_{\mathrm{AdSat}, \mathrm{BM}} * \mathrm{~L}\right) \\
& (9)
\end{aligned}
$$

So the total messaging $\operatorname{cost} \mathrm{C}_{\mathrm{MSG}}$ is

$$
\begin{aligned}
& \mathrm{C}_{\mathrm{MSG}}=\mathrm{C}_{\mathrm{MSG}, \mathrm{DAY}}+\mathrm{C}_{\mathrm{MSG}, \mathrm{HO}} \\
& =24 *\left\{\left(\mathrm{M} . \mathrm{H}_{\mathrm{Sat}, \mathrm{BM}} * 3600\right) / \mathrm{t}\right\} * \mathrm{n} \\
& +\quad \mathrm{M}^{*}\left\{\mathrm{H}_{\mathrm{CSat}, \mathrm{BM}} *(\mathrm{~K}-\mathrm{L})+\right. \\
& \mathrm{H}_{\mathrm{AdSat}, \mathrm{BM}}{ }^{* \mathrm{~L})}
\end{aligned}
$$

So the total cost of handover $\mathrm{C}_{\mathrm{Tot}}$ is

$$
\begin{aligned}
& \mathrm{C}_{\mathrm{Tot}}=\left(\mathrm{C}_{\mathrm{MSG}}+\mathrm{M} \cdot \mathrm{H}_{\mathrm{AR}, \mathrm{AR}}\right)^{*} \mathrm{R}_{\mathrm{HO}}(\mathrm{t}) \\
=\left(\left\{24 *\left\{\left(\mathrm{M} \cdot \mathrm{H}_{\mathrm{Sat}, \mathrm{BM}} * 3600\right) / \mathrm{t}\right\} * \mathrm{n}\right.\right. & +2 * \mathrm{M} * \mathrm{H}_{\mathrm{MN}, \mathrm{BM}} \\
+\mathrm{M} & \left.\left.*\left\{\mathrm{H}_{\mathrm{CSat}, \mathrm{BM}} *(\mathrm{~K}-\mathrm{L})+\mathrm{H}_{\mathrm{AdSat}, \mathrm{BM}} * \mathrm{~L}\right)\right\}+\mathrm{M} \cdot \mathrm{H}_{\mathrm{AR}, \mathrm{AR}}\right)^{*} \mathrm{R}_{\mathrm{HO}}(\mathrm{t})
\end{aligned}
$$

Equation 11 represents the total cost of algorithm based BMBHO.

\section{Simulation Result:}

In order to evaluate the performance of the new algorithm based BMBHO, we compared it to MIP \& SeaHO-LEO and the previous BMBHO. Each algorithm is evaluated by analyzing the Handoff delay, Forced call termination probability \& MN's throughput and efficiency.

The simulation results were run on MATLAB 7.8 in a designed virtual environment.

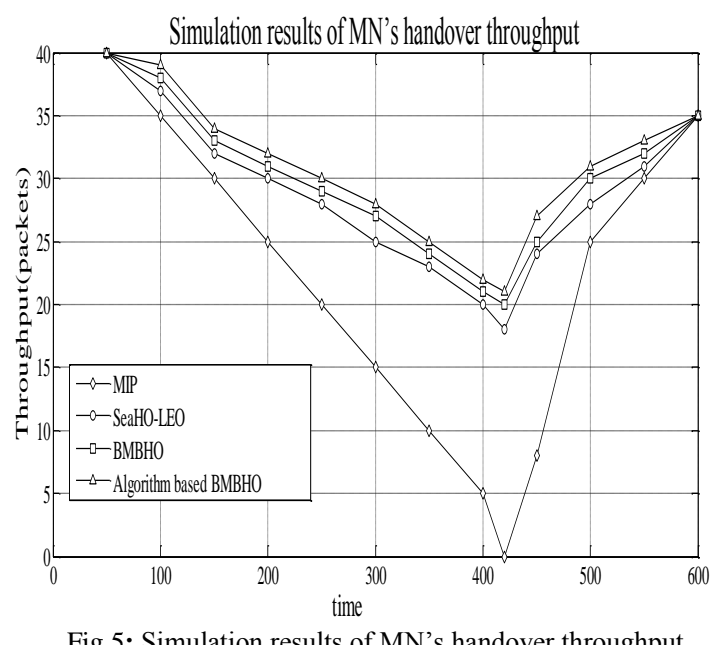

Fig 5: Simulation results of MN's handover throughput

In figure 5 we compare the Handover throughput for MIP, SeaHO-LEO \& BMBHO and algorithm based BMBHO during a handover. Due to the tunneling between HA and FA in Mobile IP network, throughput of the channel between $\mathrm{MN} 1 / \mathrm{CN}$ and $\mathrm{MN} 2 / \mathrm{MN}$ converges to zero during handover and the handover model is completed, the throughput reaches a reasonable value. SeaHO-LEO throughput is better than MIP during handover as it does not reach to zero.

In BMBHO the throughput is higher than SeaHO-LEO because the handover takes very less time and the packets during handover is sent by the old link. And here we can see that algorithm based BMBHO is far better as it has a specific algorithm to choose the best satellite for establishing connection.

In figure 6we have compared the handoff latency between the MIP,SeaHO-LEO,BMBHO and the Algorithm based BMBHO.comparing all the results we can conclude that due to ommiting the scanning process handoff delay is very less in BMBHO than the other two.

Now in the Algorithm Based BMBHO,we have taken the $\mathrm{BMBHO}$ with just an algorithm to select the satellite.so for this also handoff latency will be lesser than the MIP and SeaHO-LEO and as this finds the easiest way and lesser time to establish the connection following the specific algorithm its handoff latency is lesser than the normal BMBHO also. 


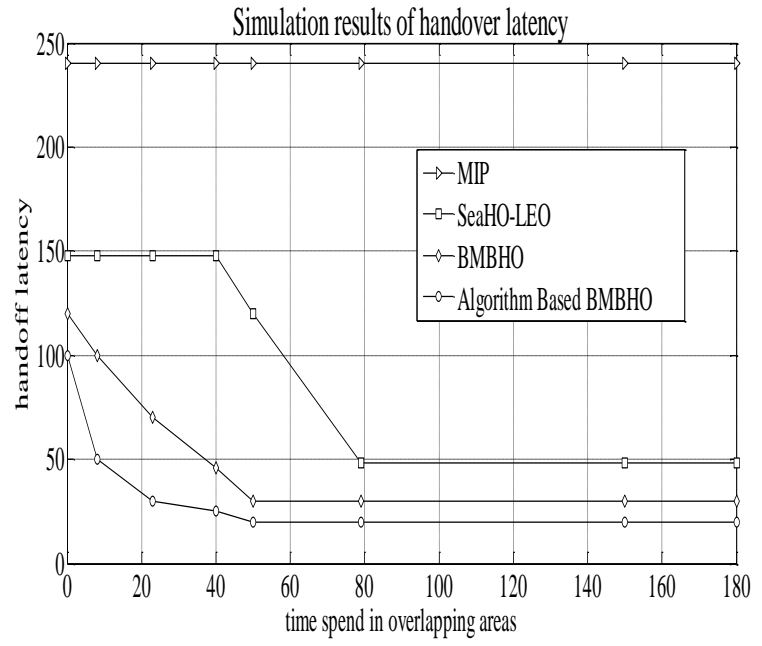

Fig 6: Simulation results of handover latency

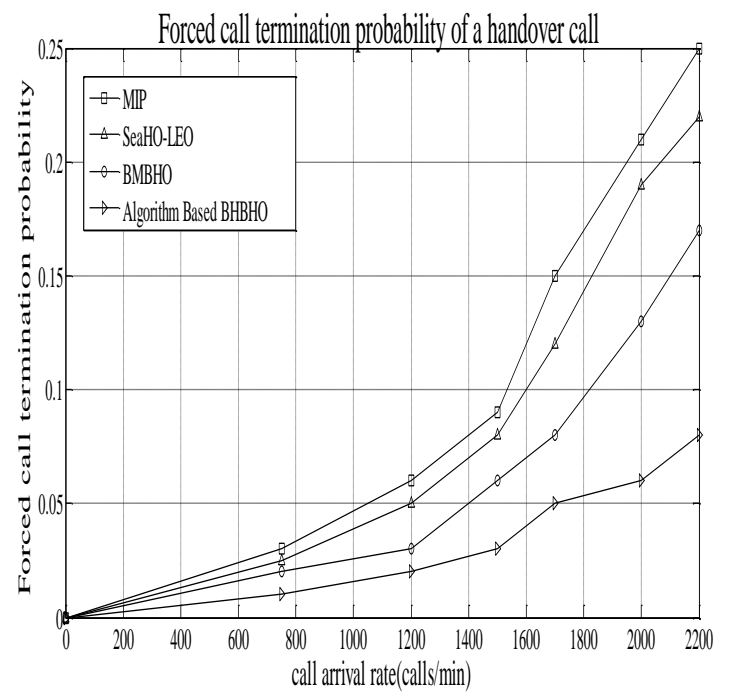

Figure7: Forced call termination probability of a handover call

In figure 7 we compare the Forced call termination probability of MIP \& SeaHO-LEO, BMBHO with the Algorithm Based BMBHO. Among these management models, BMBHO has the lowest Forced call termination probability. In MIP there is a channel allocation so MN has to wait and if there is no free channel within the handoff time the call will be terminated. In SeaHO-LEO the MN has to wait for the agent advertisement from a new satellite. If it did not received within handoff time the call is being terminated. But in BMBHO the no of channel available in the satellites seen by the $\mathrm{MN}$ at the time of handoff is already known to BM so $\mathrm{BM}$ selects the new satellite for MN which has a free channel. So the force call termination probability is reduced. Now in our approach since there is a specific algorithm for choosing the satellite. It will choose the best satellite with maximum channel capacity and for that the connection will be efficient and so is the communication and data transfer. That is why there the call termination probability is almost equal to zero. By the above result we can show that for even 2200 calls per minute the forced call termination probability is almost equal to 0.07 whereas for MIP it is 0.25 , for SeaHO-LEO it is 0.21 and for $\mathrm{BMBHO}$ it is 0.17 .

\section{ACKNOWLEDGMENT}

In this paper we have evaluated the total cost analysis of algorithm based BMBHO management where we have shown that the specific algorithm can reduce handover latency, data loss, scanning time, cost and forced call termination probability as well as can increase the MN's throughput and the efficiency.

We first described the handover is and handover process. Then we described the standard handover mechanism MIP and also SeaHO-LEO and PatHO-LEO and our BMBHO and their drawbacks. Then we have shown the specific algorithm to reduce the drawback of BMBHO. Then we have evaluated the total cost of Algorithm Based BMBHO. Relaying on the simulation results we showed that our proposed mechanism reduced handoff latency and data transfer. This algorithm can help BM to choose the best satellite for handover so that the call quality increases as well as the call dropping probability reduces to zero. Our method is more efficient than the standard one to establish the best connection.

\section{FUTURE WORK}

In future we will be focused on how to reduce the cost of Algorithm Based BMBHO in LEO satellite Networks.

\section{REFERENCES}

[1] S. L. Kota, P. A. Leppanen, and K. Pahlavan, Broadband Satellite Communications For Internet Access, Kluwer Academic Publishers, 2004.

[2] A. Jamalipour, "Satellites in IP networks," in Wiley Encyclopedia of Telecommunications, vol. 4, Wiley, 2002, pp. 2111-2122.

[3] Satellite Mobility Pattern Scheme for Centrical and Seamless Handover Management in LEO Satellite Networks Ays, eg"ulT"uys"uz and Fatih Alag" oz

[4] H. Uzunalioglu, I. F. Akyildiz, Y. Yesha, and W. Yen, "Footprint handover

[5] H. Uzunalioglu, I. F. Akyildiz, Y. Yesha, and W. Yen, "Footprint handover

Rerouting protocol for low earth orbit satellite networks," Wireless Networks, vol. 5, no. 5, pp. 327-337, 1999

[6] Systems By Joydeep Banerjee D Sarddar, S.K. Saha, M.K. Naskar, T.Jana, U. Biswas

[7] H. N. Nguyen, S. Lepaja, J. Schuringa, and H. R. Van As, "Handover management in low earth orbit satellite IP networks," IEEE Global Telecommunications Conference, San Antonio, TX, USA, pp. 27302734, 25-29 November 2001

[8] J. T. Malinen and C.Williams, "Micromobility taxonomy," Internet Draft, IETF, Nov. 2001

[9] T"uys"uz and F. Alag"oz, "Satellite mobility pattern based handover management algorithm in LEO satellites," in Proc. IEEE ICC 2006, Istanbul, Turkey, June 2006..

[10] Ays, eg"ulT"uys"uz and Fatih Alag"oz, "Satellite Mobility Pattern Scheme for centrical and Seamless Handover Management in LEO Satellite Networks", JOURNAL OF COMMUNICATIONS AND NETWORKS, VOL. 8, NO. 4, DECEMBER 2006.

[11] M. Atiquzzaman, S. Fu, and W. Ivancic, "TraSH-SN: A transport layer seamless handoff scheme for space networks," in Proc. ESTC 2004, Palo Alto, CA, June 2004.

[12] Debabrata Sarddar, Soumya Das, Dipsikha Ganguly, Sougata Chakraborty, M.k.Naskar, A New Method for Fast and Low Cost Handover in Leo Satellites(International Journal of Computer Applications (0975 - 8887) Volume 37- No.7, January 2012) http://www.ijcaonline.org/archives/volume37/number7/4622-6631 
[13] Debabrata Sarddar, Soumya Das, Dipsikha Ganguly, Sougata Chakraborty, M.k.Naskar, A New Method for Controlling Mobility Management Cost of PatHO- LEO Satellite and Mobile IP Network(International Journal of Computer Applications (0975 8887)Volume37-No.7,January2012

http://www.ijcaonline.org/archives/volume37/number7/4621-6630.

[14] Dipsikha Ganguly, Debabrata Sarddar, Soumya Das, Suman Kumar Sikdar, Sougata Chakraborty and Kunal Hui. Article: Algorithm Based Approach for the Connection Establishment in the Fast Handover in Leo Satellites in BMBHO. International Journal of Computer Applications 44(12):36-42, April 2012. Published by Foundation of Computer Science, New York, USA

[15] Debabrata Sarddar, Dipsikha Ganguly, Soumya Das, Suman Kumar Sikdar, Sougata Chakraborty, Kunal Hui, Shabnam Bandyopadhyay, Kalyan Kumar Das and Sujoy Palit. Article: Cost Analysis of Location Manager based Handover Method for LEO Satellite Networks. International Journal of Computer Applications 45(19):1-6, May 2012. Published by Foundation of Computer Science, New York, USA

[16] P. Bhagwat, C. Perkins, and S. Tripathi, "Network layer mobility: An architecture and survey," IEEE Pers. Commun., vol. 3, no. 3, pp. 54-64, June1996.

[17] A. T. Campbell, J. Gomez, S. Kim, Z. Turanyi, C.-Y. Wan, and A. Valko, Comparison of IP micro-mobility protocols," IEEE Wireless Commun. Mag., vol. 9, no. 1, Feb. 2002.

[18] H. Tsunoda, K. Ohta, N. Kato, and Y. Nemoto, "Supporting IP/LEO satellite networks by handover-independent IP mobility management," IEEE J.Select. Areas Commun., vol. 22, no. 2, pp. 300-307, 2004.

[19] Debabrata Sarddar, Dipsikha Ganguly, Soumya Das, Suman Kumar
Sikdar, Sougata Chakraborty, Kunal Hui, Shabnam Bandyopadhyay, Kalyan Kumar Das and Sujoy Palit. Article: Cost Analysis of Mobile IP for the Repetitive IP Stations during a Short Period of Time. International Journal of Computer Applications 45(19):7-12, May 2012. Published by Foundation of Computer Science, New York, USA.

[20] S. Kalyanasundaram, E.K.P Chong, and N.B. Shroff, "An efficient scheme to reduce handoff dropping in LEO satellite systems," Wireless Networks, vol. 7, no. 1, pp. 75-85, January 2001.

[21] H. N. Nguyen, S. Lepaja, J. Schuringa, and H. R. Van As, "Handover management in low earth orbit satellite IP networks," IEEE Global Telecommunications Conference, San Antonio, TX, USA, pp. 27302734, 25-29 November 2001.

\section{AUTHORS PROFILE}

Suman Kumar Sikdar is currently pursuing his $\mathrm{PhD}$ at Kalyani University . He completed his M.Tech in CSE from jadavpur University in 2011 and B-Tech in Computer Science \& Engineering from Mursidabad College of engineering and technology under West Bengal University of Technology in 2007. His research interest includes wireless communication and satellite communication. Email:sikdersuman@gmail.com

Soumya Das, son of Mr. Subrata Das and Mrs. Swapna Das, currently pursuing his B.Tech in Electronics \& Communication Engg. at Bengal Institute of Technology under West Bengal University of Technology. His research interest includes mobile communication \& satellite communication.

Debabrata Sarddar (Asst. Professor in Kalyani University) is currently pursuing his $\mathrm{PhD}$ at Jadavpur University. He completed his M.Tech in Computer Science \& Engineering from DAVV, Indore in 2006, and his B.Tech in Computer Science \& Engineering from Regional Engineering College(NIT), Durgapur in 2001. His research interest includes wireless and mobile communication_Email:dsarddar@rediffmail.com 